\section{Trombolisis mecánica y fármaco- mecánica en el tratamiento de trombosis venosa profunda sin respuesta clínica con tratamiento médico convencional}

LEOPOLDO MARINE, JESÚS URBINA, MICHEL BERGOEING, FRANCISCO VALDÉS, RENATO MERTENS, ALBRECHT KRAMER

\section{Mechanical and pharmacomechanical trombolysis in deep venous thrombosis with no clinical response to conventional treatment}

Background: Conventional treatment of deep vein thrombosis (DVT) is anticoagulation, bed rest and limb elevation. Proximal DVT patients with persisting edema, pain and cyanosis of extremities despite of conventional therapy may develop ischemia. Direct treatment of thrombosis becomes necessary. Aim: To report our experience with mechanical trombolysis of proximal lower extremity DVT. Material and Methods: Retrospective review of medical records of proximal DVT patients treated with thrombolysis between March 2012 and August 2015. Thirteen patients, 14 limbs, median age 34 years (22-85), 8 women, were admitted with pain and swelling of recent onset; one patient with venous gangrene. All patients initially received heparin in therapeutic doses without clinical improvement. Results: In all 13 cases, mechanical thrombolysis was performed using AngioJet ${ }^{\circledR}$, and associated with single dose thrombolytic agent in 9. Additional angioplasty for residual stenosis was performed in 12 (7 stents) and IVCF were implanted in 8 . All patients were subsequently anticoagulated. Early outcomes with disappearance of pain and decrease of edema, with no mortality or bleeding complications. The patient with foot gangrene required amputation. Conclusions: Mechanical thrombolysis with a single dose of a thrombolytic agent is safe and effective in patients with proximal DVT with an unfavorable evolution.

(Rev Med Chile 2017; 145: 63-71)

Key words: Gangrene; Mechanical Thrombolysis; Thrombolytic Therapy; Thrombophlebitis; Venous Thrombosis.

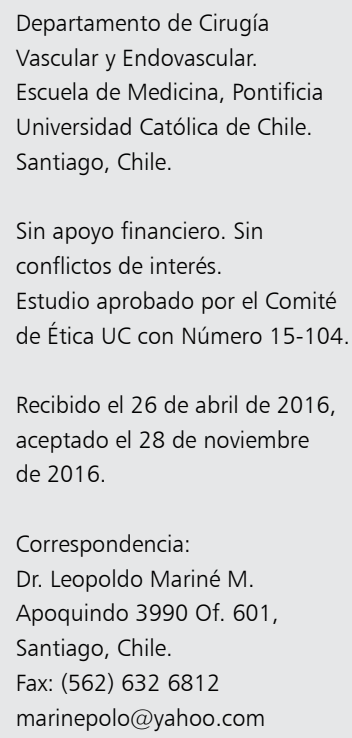

L a trombosis venosa profunda (TVP) proximal o ilio-femoral es un subgrupo de TVP con mayor riesgo de complicaciones agudas, como el tromboembolismo pulmonar (TEP) o la flegmasia ${ }^{1}$, y crónicas como el síndrome post-trombótico $(\mathrm{SPT})^{1-3}$ y la recurrencia deTVP ${ }^{4}$.

El tratamiento convencional inicial de la TVP es la anticoagulación y el reposo absoluto con elevación de la extremidad comprometida (posición de Trendelenburg). Pacientes con TVP proximales que persisten con edema, dolor y cianosis de la extremidad pese a tratamiento convencional pueden evolucionar a isquemia. En ellos es necesario un tratamiento directo sobre la trombosis ${ }^{1,5,6}$ para 
permitir el rápido alivio de la obstrucción venosa, lo que ha demostrado disminuir el daño valvular, el SPT y la recurrencia de TVP5.

El concepto de "vena abierta" es una estrategia activa de eliminación rápida de la obstrucción venosa por extracción, lisis y/o aspiración del trombo. Intervenciones como la trombectomía venosa $^{7}$ y la trombolisis dirigida por catéter ${ }^{1,5}$ se han realizado en el pasado con morbilidad relevante. La trombolisis fármaco-mecánica (TFM) ha surgido en los últimos años como una alternativa efectiva para el tratamiento de la TVP proximal con evolución desfavorable ${ }^{5}$.

Presentamos nuestra experiencia con trombolisis mecánica $(\mathrm{TM})$ y fármaco-mecánica en pacientes con TVP proximal que presentan falta de respuesta clínica al tratamiento médico convencional o amenaza de gangrena venosa.

\section{Material y Método}

Estudio retrospectivo de 13 pacientes consecutivos portadores de TVP extensa con o sin flegmasia, intervenidos con TM o TFM entre marzo de 2012 y agosto de 2015, siguiendo una metodología de tratamiento similar. Todos los pacientes presentaban TVP proximal y 9 adicionalmente TVP fémoro-poplitea. Se revisan los registros clínicos, imágenes diagnósticas y seguimiento clínico con clasificación $\mathrm{CEAP}^{8}$ y Escala Villalta ${ }^{9}$ imagenológico.

El diagnóstico clínico inicial de TVP se confirmó mediante dúplex venoso. Los pacientes fueron tratados inicialmente con reposo absoluto en posición de Trendelenburg y heparina no fraccionada endovenosa en dosis terapéutica efectiva controlada ${ }^{10}$. Los pacientes que consultaron con flegmasia o aquellos que cursando con TVP proximal evolucionan sin mejoría clínica a pesar del tratamiento médico señalado, fueron seleccionados para manejo con TFM. La ausencia de mejoría clínica se definió como la persistencia de edema significativo y dolor de la extremidad luego de $48 \mathrm{~h}$ de tratamiento médico controlado.

La trombolisis fue planificada en base a características clínicas e imágenes del angio-TAC y siguiendo los criterios de selección señalados en la Tabla 1.

Pacientes con mayor riesgo de hemorragia fueron incluidos, pero tratados solo con TM. Se definió como éxito angiográfico la lisis de $\geq 50 \%$ de la trombosis comparado con la flebografía inicial ${ }^{11}$ y como éxito clínico, la desaparición del dolor, recuperación de la viabilidad de la extremidad en caso de flegmasia o la disminución del edema y congestión de la extremidad.

\section{Metodología de tratamiento en TM y TFM}

1. Intervención en sala de angiografía o híbrida, bajo anestesia local con sedación o anestesia general (Figura 1).

2. Instalación de filtro de vena cava inferior (FVCI) por vía yugular o femoral contralateral para profilaxis de TEP.

3. Acceso por punción eco-guiada de vena permeable caudal al sitio de la trombosis, utilizándose la vena femoral o safena interna en TVP ilio-cava o la vena poplítea si es fémoro-poplitea.

4. Flebografía inicial para confirmar la extensión de la trombosis y definir el trayecto a seguir con las guías y catéteres.

5. TFM con AngioJet ${ }^{\circledR}$ (Boston Scientific, EE. UU.) que se realiza en intervención única en tres fases: a) Trombolisis-trombectomía mecánica inicial en que se realiza maceración-aspiración del trombo; b) Trombolisis farmacológica mediante la administración de activador recombinante del plasminógeno tisular (rTPA o Alteplase, Actilyse ${ }^{\circledR}$ Boehringer

Tabla 1. Criterios de selección de pacientes

\begin{tabular}{|ll|}
\hline Criterios de inclusión & Criterios de exclusión \\
\hline $\begin{array}{l}\text { - TVP proximal sin mejoría clínica luego de } 48 \mathrm{~h} \text { de trata- } \\
\text { miento médico convencional }\end{array}$ & $\begin{array}{l}\text { TVP proximal con mejoría clínica con tratamiento médico } \\
\text { convencional }\end{array}$ \\
\hline - Tiempo de evolución de TVP $\leq 15$ días & $\begin{array}{l}\text { Tiempo de evolución de TVP proximal de }>15 \text { días en } \\
\text { ausencia de flegmasia }\end{array}$ \\
- Flegmasia cerúlea independiente de tiempo de evolución & - Estenosis venosas llio-femorales sin TVP \\
\hline
\end{tabular}




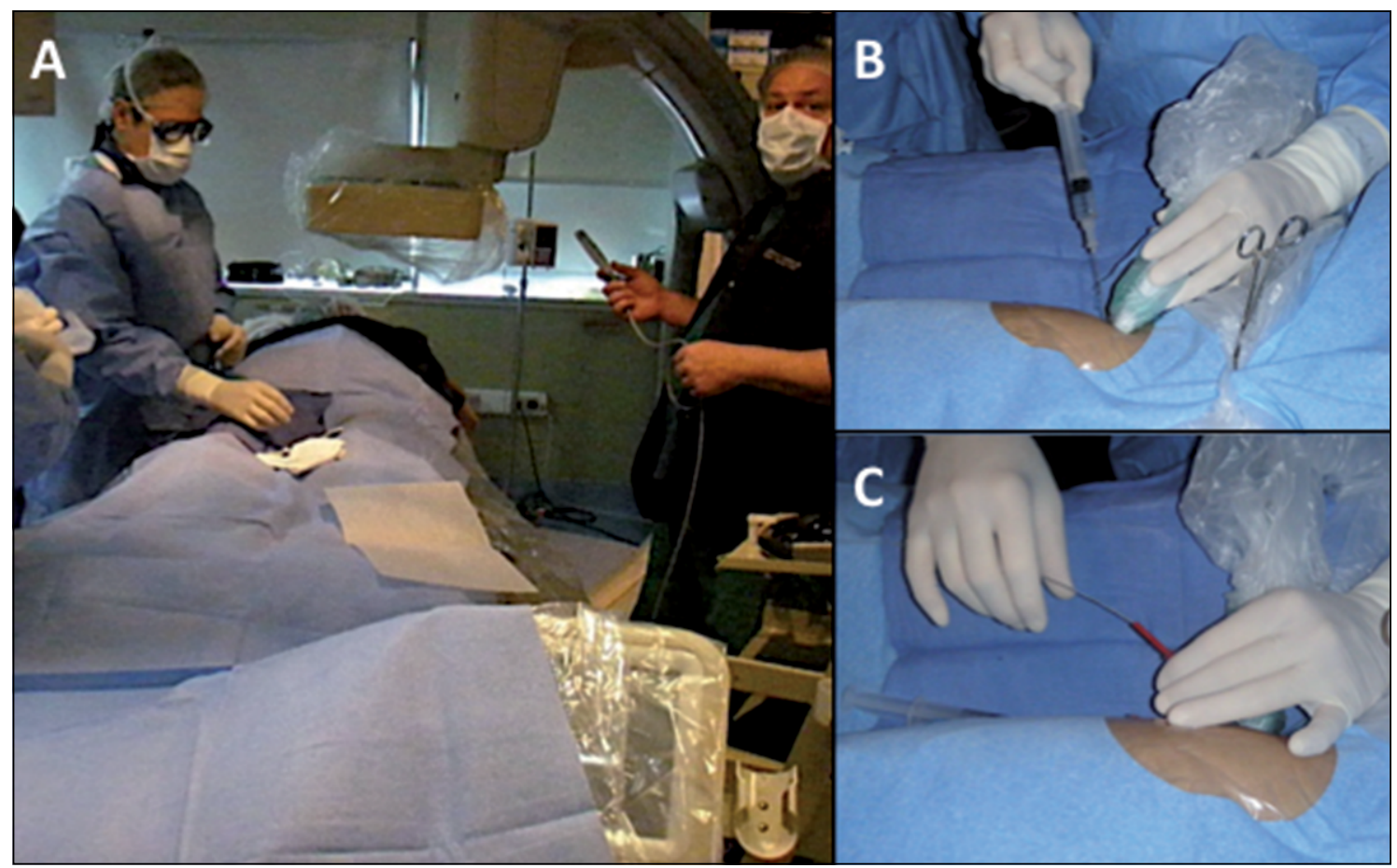

Figura 1. Imágenes de la intervención de trombolisis en pabellón de angiografía. En A se observa a paciente en posición decúbito ventral, con anestesia general. El anestesista tiene probe de ecografía en su mano, próximo a entregarlo para su preparación y uso por el cirujano. En B se observa punción eco-guiada de vena poplítea. En C se observa el avance de la guía para la colocación posterior de la vaina.

Ingelheim, Alemania) en dosis de hasta $10 \mathrm{mg}$ con técnica de inyección pulsada a alta presión (Técnica Power-Pulse); c) Luego de 20 min de incubación del trombolítico, se realiza nuevamente trombolisis-trombectomía mecánica con modalidad maceración-aspiración hasta un máximo de $12 \mathrm{~min}$. En la TM exclusiva no se administra rTPA.

6. Control flebográfico durante la trombolisis y al final de esta.

7. Angioplastía con o sin implante de stent, complementarias en caso de estenosis residual o trombo antiguo no recanalizable por la trombolisis.

8. Retiro del acceso y hemostasia por compresión del sitio de punción.

9. Anticoagulación con heparina endovenosa intra y post-operatoria, luego anticoagulación oral con cumarínicos o rivaroxaban post-alta, acompañado de compresión elástica graduada de la extremidad.

10. Control y seguimiento.

\section{Resultados}

Las características clínicas de los 13 pacientes tratados se describen en las Tablas 2 y 3.

La edad fue de 34 años (mediana, rango: 22$85), 8$ pacientes mujeres $(61,5 \%)$. Se trataron 14 extremidades, 7 izquierdas, 5 derechas y una bilateral. La duración de los síntomas previos a la intervención fue de 7 días (mediana, rango: 1-29). Tres pacientes presentaban flegmasia cerúlea dolens, uno de ellos con cáncer pulmonar metastásico que ingresó con gangrena del pie (Figura 2).

En una paciente con cáncer cérvico-uterino, que ingresó con flegmasia cerúlea dolens, no se obtuvo angio-TAC pre tratamiento dada la urgencia terapéutica. En 6 pacientes $(46,2 \%)$ la angio-TAC de tórax permitió realizar el hallazgo diagnóstico de TEP asintomático concomitante. En un paciente se diagnosticó hipoplasia de la vena cava inferior (VCI) infrarrenal con trombosis de esta y de la red venosa colateral compensatoria (venas ácigos y hemi-ácigos). Adicionalmente, en todos 
Tabla 2. Condiciones subyacentes responsables de la extensión de la trombosis (n pacientes, \%)

\begin{tabular}{|ll|}
\hline Síndrome de May-Thurner & $4(30,8 \%)$ \\
\hline Cáncer & $3(23,1 \%)$ \\
Ca Colon Metastásico & 1 \\
Ca Cérvico-Uterino metastásico & 1 \\
Ca Pulmonar metastásico & 1 \\
Trombosis de FVCI previo & $3(23,1 \%)$ \\
Bajo nivel del filtro & 2 \\
Sobre nivel del filtro & 1 \\
Trombofilia asociada & 2 \\
\hline Hipoplasia de vena cava Inferior & $1(7,7 \%)$ \\
\hline Colitis ulcerosa & $1(7,7 \%)$ \\
No establecida & $1(7,7 \%)$ \\
\hline
\end{tabular}

Tabla 3. Formas de Presentación clínica (n pacientes, \%)

\begin{tabular}{|lc|}
\hline Trombosis venosa iliofemoral & 13 (100\%) \\
Extensión a VCI & 7 \\
Trombosis femoral & 11 \\
\hline Flegmasia Cerúlea Dolens & $3(23,1 \%)$ \\
Ca colon metastásico & 1 \\
Ca pulmonar metastásico & 1 \\
Colitis ulcerosa & 1 \\
Tromboembolismo pulmonar & $6(46,2 \%)$ \\
Ca colon metastásico & 1 \\
Ca pulmonar avanzado & 1 \\
Colitis ulcerosa & 1 \\
Trombosis sobre FVCI & 1 \\
Uso de estrógenos & 1 \\
May-Thurner & 1 \\
\hline
\end{tabular}

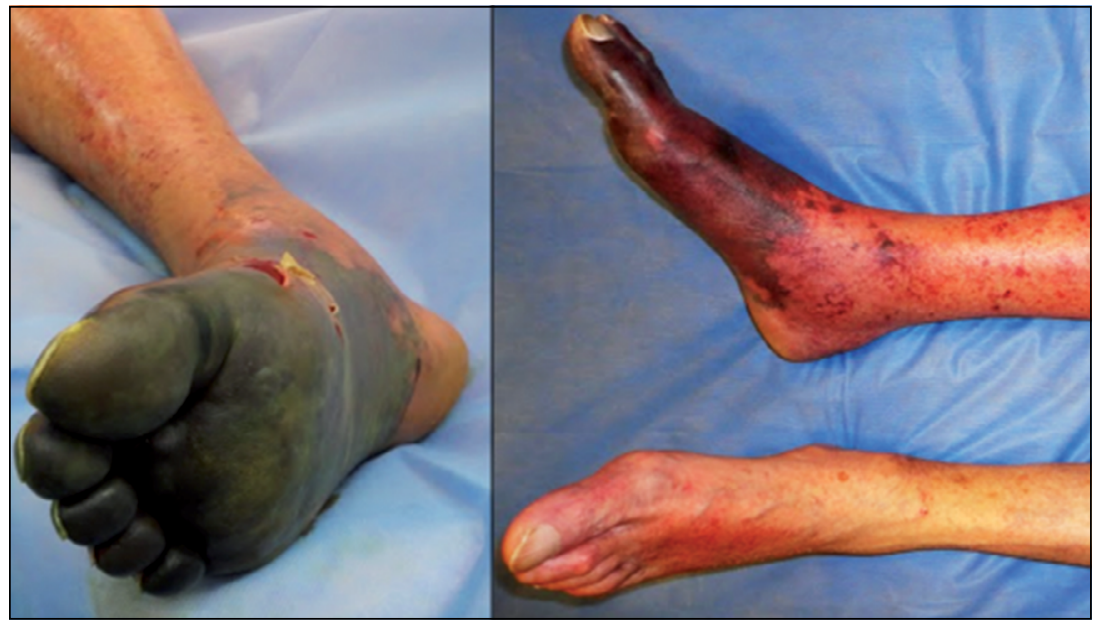

Figura 2. Imágenes de gangrena venosa. Paciente con cáncer pulmonar metastásico que consulta con flegmasía cerúlea dolens de extremidad inferior derecha y gangrena venosa. Se observa necrosis de antepie en imágenes capturadas desde proyección plantar (izquierda) y lateral (derecha). los pacientes no neoplásicos se realizó estudio de trombofilia, siendo anormal en 5 de 10 pacientes estudiados (50\%): síndrome antifosfolípido en 3 casos, déficit de antitrombina III ( 1 caso) y déficit de proteína C (1 caso). Dos pacientes con trombofilia presentaban síndrome de May-Thurner.

En la Tabla 4 se detalla el tratamiento efectuado utilizando el sistema AngioJet ${ }^{\circledR}$. Se realizó acceso de la vena poplítea en 10 pacientes $(76,9 \%)$, por vena femoral en dos pacientes (uno bilateral) y por punción de vena safena interna cercano a su cayado en otra.

En 7 pacientes se instaló un FVCI removible Optease ${ }^{\circledR}$ (Cordis, Estados Unidos) y en un caso filtro bioconvertible Sentry ${ }^{\circledR}$ (Novate, Irlanda).
Cinco pacientes tenían FVCI implantados previamente. Un caso requirió un segundo FVCI suprarrenal Optease ${ }^{\circledR}$ (Cordis, Estados Unidos) por trombosis parcial protruyente del filtro previo. El paciente de la hipoplasia de VCI no presentaba riesgo de embolía pulmonar por lo que no requirió FVCI.

En 9 pacientes utilizamos rTPA. En 4 pacientes no se administró trombolítico por riesgo de hemorragia: dos de los casos con neoplasias metastásicas (colon, cérvico-uterino), un paciente de trombosis de FVCI que cursaba con trombosis de seno venoso intracraneano y otro de síndrome de May-Thurner sometida a una biopsia endoscópica reciente. 
Tabla 4. Características de la Intervención (n pacientes, \%)

\begin{tabular}{|c|c|}
\hline Filtro de vena cava inferior & $12(92,3 \%)$ \\
\hline Previo a TFM & 5 \\
\hline En pabellón & 8 \\
\hline Sin filtro & 1 \\
\hline \multicolumn{2}{|l|}{ Anestesia } \\
\hline Local y sedación & 8 \\
\hline General & 5 \\
\hline \multicolumn{2}{|l|}{ Acceso } \\
\hline Poplíteo & 9 \\
\hline Femoral & 4 \\
\hline $\begin{array}{l}\text { Trombolisis mecánica } \\
\text { Tiempo de trombolisis: } 508,2 \pm 130,2 \\
(270-720) \text { segundos }\end{array}$ & $13(100 \%)$ \\
\hline Trombolisis farmacológica & $9(69,2 \%)$ \\
\hline \multicolumn{2}{|l|}{ Dosis: 5-10 mg (mediana 10 mg) } \\
\hline Tratamiento endovascular complementario & $12(92,3 \%)$ \\
\hline Angioplastía sola (6-14 mm) & 5 \\
\hline Angioplastía y stent (6-16 mm) & 7 \\
\hline Sin angioplastía/stent & 1 \\
\hline
\end{tabular}

Completada la trombolisis, 12 pacientes requirieron angioplastía complementaria por estenosis residual mayor de 50\% (Figura 3), desplegándose stent ilíaco o ilio-cavo adicional en 7 de ellos al presentar estenosis residual post-angioplastia. Los 4 casos de síndrome de May-Thurner fueron tratados primariamente con stents, de preferencia autoexpansibles (Tabla 4). No hubo sangrado significativo asociado ni necesidad de fasciotomía posterior.

Once pacientes fueron observados en Unidad de Recuperación por $16 \mathrm{~h}$ (mediana, rango: 6-77,5 h), 2 se trasladaron directo a su habitación. La estada hospitalaria fue de 6 días (mediana, rango: 3-16). En 4 pacientes (30,8\%) fue necesario transfundir glóbulos rojos, en promedio 3,5 unidades por paciente, tres de ellos pacientes oncológicos y una paciente con anemia crónica previa post-bypass gástrico. Cuatro casos presentaron hemoglobinuria transitoria sin deterioro de la función renal. Cinco pacientes fueron transfundidos (mediana 2 unidades de glóbulos rojos).

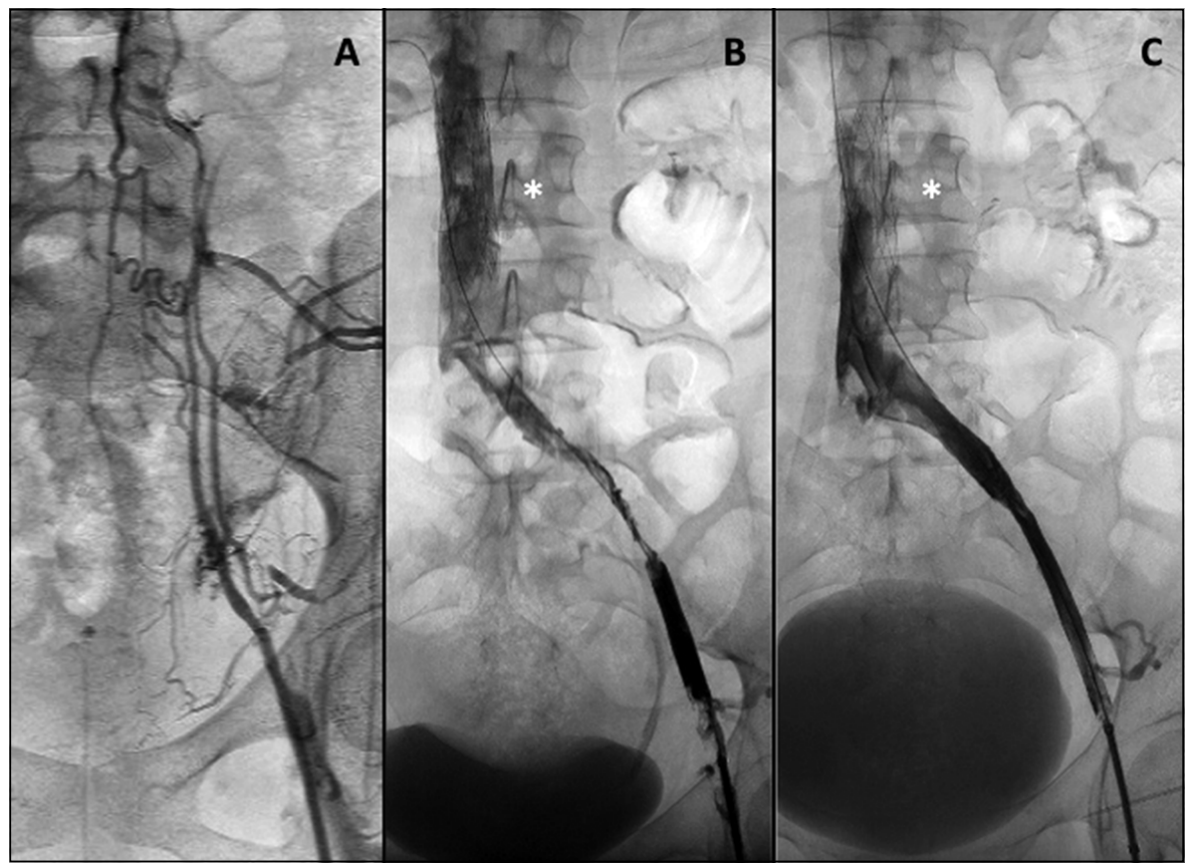

Figura 3. Imágenes de estudios angiográficos. Paciente de 34 años con TVP ilíaca de extremidad inferior izquierda y persistencia de edema y dolor luego de tratamiento anticoagulante habitual. En $\mathbf{A}$ la flebografía inicial realizada por acceso femoral izquierdo muestra oclusión de eje ilíaco venoso izquierdo con red venosa colateral. La imagen B muestra los resultados de la trombolisis fármaco-mecánica con 10 mg de rTPA, observándose mejor permeabilidad de la vena, pero advirtiéndose la presencia de estenosis significativa en la vena ilíaca externa izquierda. En C se observa resultado final luego de angioplastía con balón de 12 × 20 mm, sin estenosis residual, por lo que no fue necesaria la colocación de stent. 
Tabla 5. Resultados y seguimiento (n pacientes, \%)

\begin{tabular}{|lc|}
\hline Mortalidad intrahospitalaria & 0 \\
\hline Amputación mayor intrahospitalaria & $1(7,7 \%)$ \\
\hline Morbilidad intrahospitalaria relacionada & $6(46,2 \%)$ \\
Hemoglobinuria & 4 \\
Hemorragia digestiva baja & 1 \\
\hline Transfusión & $4(30,8 \%)$ \\
Anticoagulación/antiagregación al alta & $13(100 \%)$ \\
Warfarínicos & 1 \\
Cumarínicos & 6 \\
Nuevos anticoagulantes & 4 \\
HBPM & 2 \\
Aspirina & 6 \\
Clopidogrel & 2 \\
Promedio de seguimiento & $31,4 \pm 15,3$ \\
& meses \\
Mortalidad en el seguimiento & $3(23,1 \%)$ \\
Ca colon metastásico & 4 meses \\
Ca cérvico-uterino metastásico & 3 meses \\
Ca pulmonar metastásico & 1 mes \\
\hline
\end{tabular}

No hubo reintervención. No hubo mortalidad ni TEP clínico (Tabla 5). Todos presentaron éxito angiográfico y clínico, con desaparición del dolor y disminución importante del edema. Sólo el caso con gangrena del pie requirió amputación supracondílea, logrando su cicatrización mediante el tratamiento de trombolisis. Las lesiones isquémicas en los otros casos oncológicos evolucionaron con regresión.

Los pacientes fueron dados de alta con tratamiento anticoagulante y/o antiagregante (Tabla 5) y soporte elástico graduado de 20-30 $\mathrm{mm} \mathrm{Hg}$ en el tobillo. En 3 casos de síndrome de May-Thurner, los FVCI fueron retirados dentro del mes después de la trombolisis, la cuarta paciente rechazó retiro.

El seguimiento promedio fue de 33,6 $\pm 14,9$ (rango: 9,3-49,5) meses. Los 3 pacientes con cáncer fallecieron por su enfermedad original entre 1 y 4 meses, sin recurrencia de la flegmasia. En el seguimiento alejado con imágenes, 2 casos presentan trombosis venosa, sin repercusión clínica: la paciente con TVP bilateral y déficit de antitrombina III, se encuentra asintomática, sin edema residual a los 2 años, y una paciente con TVP ilio-femoral tratada con angioplastía exclusiva, asintomática por más de 1 año. El seguimiento clínico con CEAP muestra una mediana de 2 , siendo C0 (30\%), C1 (10\%), C2 (30\%), C3 (10\%), C4 (20\%) y agrupados C0-C3 80\%. La Escala Villalta mostró una mediana de 2, con score de 0 (30\%), 1 (10\%), $2(30 \%)$ y $3(30 \%)$, que corresponden a ausencia de insuficiencia venosa profunda.

\section{Discusión}

El manejo conservador de las TVP proximales con reposo absoluto, posición de Trendelenburg y anticoagulación endovenosa con heparina sódica terapéutica o heparina de bajo peso molecular (HBPM) es eficaz en la mayoría de los casos no complicados $^{12}$. Los pacientes con TVP proximal que evolucionan clínicamente en forma desfavorable con tratamiento médico convencional o que consultan con flegmasia o gangrena venosa deben ser estudiados con angio-TAC de abdomen y pelvis en fase venosa e iniciar un tratamiento directo y activo sobre la trombosis. El estudio con imágenes debe ser precoz, ya que permite obtener información del límite de extensión proximal, el compromiso de red venosa colateral, co-existencia de TEP (30\% de los casos) ${ }^{13} \mathrm{y}$, eventualmente, la causa subyacente (cáncer o compresión extrínseca) ${ }^{13}$.

El manejo activo tiene por objetivo restablecer rápidamente la permeabilidad venosa ${ }^{13}$, así como reducir la carga de trombo, lo que permite aliviar el dolor y el edema ${ }^{14}$. Dentro de las alternativas de manejo conocidas están la trombectomía venosa $^{7}$, la trombolisis dirigida por catéter ${ }^{1,5}$ y la $\mathrm{TFM}^{5,13,14}$. La trombectomía venosa fue utilizada en el pasado para el tratamiento de la flegmasia cerúlea dolens, pero presenta inconvenientes de pérdida sanguínea significativa, imposibilidad de limpiar trombos de venas pequeñas, daño valvular frecuente (SPT) y alta incidencia de retrombosis ${ }^{15}$. Posteriormente, el desarrollo de la trombolisis dirigida por catéter ha permitido la administración directa de trombolíticos en el sitio de la trombosis a través de acceso percutáneo distante, con menor SPT y mejores resultados comparados con el tratamiento anticoagulante exclusivo ${ }^{5}$. Su mayor limitación es el prolongado tiempo de repermeabilización venosa (tarda $54-55$ h promedio) ${ }^{1,16}$, lo que incrementa el riesgo de hemorragias severas (1-11\%) e intracraneana hasta $0,2 \%{ }^{17,18}$.

La TFM se ha desarrollado en los últimos años como una alternativa a la trombolisis dirigida por catéter, que permite remover el trombo en una sola intervención que dura de 1-3 h, obviando la necesidad de infusión sostenida de trombolíticos 
posteriormente ${ }^{5}$, preservar la función valvular y realizar el diagnóstico y tratamiento de lesiones anatómicas subyacentes por vía endovascular inmediata $^{19,20}$. Combina la administración local del trombolítico con un mecanismo físico que permiten la mejor dispersión de la droga, la maceración del trombo y su aspiración posterior por efecto Venturi. La técnica "lisis y esperar" que utilizamos en nuestros pacientes con el sistema AngioJet ${ }^{\circledR}$ permite que el agente lítico "bañe" y degrade al trombo durante 20 a 45 min antes de la trombectomía mecánica ${ }^{20}$. En casos de contraindicación absoluta de uso de trombolíticos, realizamos TM exclusiva, sin la administración de fármacos, como ha sido descrito anteriormente por otros ${ }^{21}$.

En comparación con la trombolisis dirigida por catéter, se logra una reducción de la dosis de trombolítico $^{22}$ y de su tiempo de administración, lo que permite repermeabilizar segmentos trombosados en forma más rápida y segura, con menor estada en UCI, menos días de hospitalización, menor necesidad de transfusión y mejor costo-efectividad ${ }^{19-23}$; presentando resultados terapéuticos similares a los de la trombolisis dirigida por catéter, pero con una reducción en las complicaciones hemorrágicas ${ }^{24}$. Los buenos resultados de la TFM reportados en series no prospectivas ${ }^{16,21,23}$ la han hecho recomendable por las guías de manejo de TVP de distintas sociedades científicas como la primera línea de tratamiento en TVP ilio-femorales seleccionadas $^{6,25-29}$, en especial ante flegmasia cerúlea dolens ${ }^{14}$, trombosis aguda de la VCI, progresión proximal rápida a pesar de anticoagulación, trombosis de FVCI y en TVP extensas con ausencia de mejoría de síntomas ${ }^{5,26-28}$, como es el caso de la mayoría de los pacientes de esta serie. Estudios prospectivos en curso permitirán aclarar mejor las indicaciones de tratamiento en el futuro ${ }^{5}$.

Después de completar la TFM es frecuente observar trombo residual antiguo o desenmascarar una obstrucción venosa subyacente, que requieren de angioplastía, con o sin stent, para restablecer un adecuado flujo para prevenir la retrombosis y aumentar la permeabilidad ${ }^{30}$. La evidencia disponible aconseja que la angioplastia se acompañe de stent para prevenir reestenosis elástica inmediata o recoil, aliviar compresión externa y establecer adecuado flujo de salida ${ }^{31}$. La mayoría de nuestros pacientes requirieron tratamiento complementario, 2/3 con necesidad de stents ${ }^{4,19}$, en especial en casos de síndrome de May-Thurner para evitar el recoil por compresión externa ${ }^{28}$. Es curioso observar que la buena respuesta clínica que tienen estos tratamientos no se correlacionan con respuesta venográfica ${ }^{3}$, lo que ocurre también en el seguimiento, como hemos observado en el caso descrito, posiblemente por reclutar ramas colaterales ocluidas.

Las complicaciones por TFM son infrecuentes, siendo descritas hemólisis, hemorragias mayores (2-4\%) y TEP secundario por desplazamiento del trombo. Esto último hace recomendar el uso rutinario de FVCI transitorios o permanentes ${ }^{4,7,19,20,23}$. Todos nuestros pacientes estaban protegidos de TEP durante la intervención y el retiro de FVCI se realizó solamente en 3 casos de síndrome de May-Thurner. La principal complicación alejada es la reoclusión, por lo que es importante que el paciente permanezca con tratamiento anticoagulante prolongado o eventualmente de por vida, si alguna condición subyacente así lo indica, y use, además, soporte elástico graduado para prevención del $\mathrm{SPT}^{2}$.

Los casos de TVP proximal que evolucionen a flegmasia cerúlea dolens deben manejarse más agresivamente y en forma más precoz ( 6 a 12 h) para evitar la pérdida de la extremidad ${ }^{12}$. Las condiciones protrombogénicas subyacentes en la población de este estudio (Tabla 2) son semejantes a las publicadas para la flegmasia cerúlea dolens ${ }^{13}$. Los factores adversos en la evolución de flegmasia cerúlea dolens son: cáncer ${ }^{13}, \mathrm{TEP}^{13}$ y gangrena venosa al momento de consultar ${ }^{12}$, en especial si requiere amputación mayor ${ }^{15}$. Esta ha sido también nuestra experiencia en el paciente que se presentó con gangrena, cuyo desenlace es similar al publicado por Erdoes et al. ${ }^{32}$. Aunque no cambie el pronóstico global en los pacientes con cáncer, el tratamiento trombolítico permite realizar salvataje de extremidades, como ocurrió en el resto de los pacientes de esta serie.

Otro factor a considerar es el tiempo de duración de la TVP, siendo mejores los resultados en procesos menores a10-14 días de evolución ${ }^{25}$. El paciente con gangrena se trató por TVP de 29 días, el resto de la serie presentaba menos de 15 días de evolución.

\section{Conclusión}

Los resultados favorables de la TFM y TM en esta serie se sustentan en los beneficios de un 
tratamiento mínimamente invasivo, realizado en intervención única, con buen éxito técnico, de baja morbilidad asociada y con posibilidades de complementarse con otras modalidades de tratamiento endovascular. En casos de TVP proximales que no responden a tratamiento médico habitual puede plantearse como una alternativa terapéutica en etapas precoces, aun en casos de gangrena venosa.

\section{Referencias}

1. Amin VB, Lookstein RA. Catheter-directed interventions for acute iliocaval deep vein thrombosis. Tech Vasc Interv Radiol 2014; 17 (2): 96-102.

2. Prandoni $P$, Lensing AW, Prins MH, Frulla M, Marchiori A, Bernardi E, et al. Below-knee elastic compression stockings to prevent the post-thrombotic syndrome. Ann Intern Med 2004; 141 (4): 249-56.

3. Tardy B, Moulin N, Mismetti P, Decousus H, Laporte $\mathrm{S}$. Intravenous thrombolytic therapy in patients with phlegmasia caerulea dolens. Haematologica 2006; 91 (2): 281-2.

4. Gasparis AP, Labropoulos N, Tassiopoulos AK, Phillips B, Pagan J, Cheng Lo, et al. Midterm follow-up after pharmacomechanical thrombolysis for lower extremity deep venous thrombosis. Vasc Endovascular Surg 2009; 43 (1): 61-8.

5. Popuri RK, Vedantham S. The role of thrombolysis in the clinical management of deep vein thrombosis. Arterioscler Thromb Vasc Biol 2011; 31 (3): 479-84.

6. Hirsh J, Guyatt G, Albers G, Harrington R, Schunemann H. Antithrombotic and thrombolytic therapy: American College of Chest Physicians evidence-based clinical practice guidelines (8th edition). Executive summary: American College of Chest Physicians Evidence-Based Clinical Practice Guidelines (8th Edition). Chest 2008; 133 (6 suppl): 71S-109S.

7. Comerota AJ, Paolini D. Treatment of acute iliofemoral deep venous thrombosis: a strategy of thrombus removal. Eur J Vasc Endovasc Surg 2007; 33 (3): 351-60.

8. Eklöf B, Rutherford RB, Bergan JJ, Carpentier PH, Gloviczki P, Kistner RL, et al. Revision of the CEAP classification for chronic venous disorders: consensus statement. J Vasc Surg 2004; 40 (6): 1248-52.

9. Villalta S, Bagatella P, Piccioli A, Lensing A, Prins M, Prandoni P. Assessment of validity and reproducibility of a clinical scale for the postthrombotic syndrome. Hemostasis 1994; 24: 158a.

10. Mariné L, Sánchez G, Vargas JF, Zúñiga P, Aizman A, Mertens R, et al. Correlación de valores de TTPa con anti factor Xa para establecer rango terapéutico en tra- tamiento anticoagulante con heparina sódica. Rev Med Chile 2014; 142 (11): 1392-7.

11. Hager E, Yuo T, Avgerinos E, Naddaf A, Jeyabalan G, Marone L, et al. Anatomic and functional outcomes of pharmacomechanical and catheter-directed thrombolysis of iliofemoral deep venous thrombosis. J Vasc Surg Venous Lymphat Disord 2014; 2 (3): 246-52.

12. Weaver FA, Meacham PW, Adkins RB, Dean RH. Phlegmasia cerulea dolens: therapeutic considerations. South Med J 1988; 81 (3): 306-12.

13. Chinsakchai K, Ten Duis K, Moll FL, de Borst GJ. Trends in management of phlegmasia cerulea dolens. Vasc Endovascular Surg 2011; 45 (1): 5-14.

14. Chaer RA, Dayal R, Lin SC, Trocciola S, Morrissey NJ, McKinsey J, et al. Multimodal therapy for acute and chronic venous thrombotic and occlusive disease. Vasc Endovascular Surg 2005; 39 (5): 375-80.

15. Perkins JM, Magee TR, Galland RB. Phlegmasia caerulea dolens and venous gangrene. Br J Surg 1996; 83 (1): 1923.

16. Kasirajan K, Gray B, Ouriel K. Percutaneous AngioJet thrombectomy in the management of extensive deep venous thrombosis. J Vasc Interv Radiol 2001; 12 (2): 179-85.

17. Bashir R, Zack CJ, Zhao H, Comerota AJ, Bove AA. Comparative outcomes of catheter-directed thrombolysis plus anticoagulation vs anticoagulation alone to treat lower-extremity proximal deep vein thrombosis. JAMA Intern Med 2014; 174 (9): 1494-501.

18. Tung CS, Soliman PT, Wallace MJ, Wolf JK, Bodurka DC. Successful catheter-directed venous thrombolysis in phlegmasia cerulea dolens. Gynecol Oncol 2007; 107 (1): 140-2.

19. Lin PH, Zhou W, Dardik A, Mussa F, Kougias P, Hedayati $\mathrm{N}$, et al. Catheter-direct thrombolysis versus pharmacomechanical thrombectomy for treatment of symptomatic lower extremity deep venous thrombosis. Am J Surg 2006 (6); 192: 782-8.

20. Cynamon J, Stein EG, Dym J, Jagust MB, Binkert CA, Baum RA. A new method for aggressive management of deep vein thrombosis: Retrospective study of the power pulse technique. J Vasc Interv Radiol 2006; 17 (6): 10439.

21. Rao AS, Konig G, Leers SA, Cho J, Rhee RY, Makaroun MS, et al. Pharmacomechanical thrombectomy for iliofemoral deep vein thrombosis: an alternative in patients with contraindications to thrombolysis. J Vasc Surg 2009; 50 (5): 1092-8.

22. Martínez-Trabal JL, Comerota AJ, LaPorte FB, Kasanjian S, DiSalle RS, Sepanski DM. The quantitative benefit of isolated, segmental, pharmacomechanical 
thrombolysis (ISPMT) for iliofemoral venous thrombosis. J Vasc Surg 2008; 48 (6): 1532-7.

23. Bush RL, Lin PH, Bates JT, Mureebe L, Zhou W, Lumsden AB. Pharmacomechanical thrombectomy for treatment of symptomatic lower extremity deep venous thrombosis: Safety and feasibility study. J Vasc Surg 2004; 40 (5): 965-70.

24. Lin PH, Ochoa LN, Duffy P. Catheter-directed thrombectomy and thrombolysis for symptomatic lower-extremity deep vein thrombosis: review of current interventional treatment strategies. Perspect Vasc Surg Endovasc Ther 2010; 22 (3): 152-63.

25. Meissner MH, Gloviczki P, Comerota AJ, Dalsing MC, Eklof BG, Gillespie DL, et al. Society for Vascular Surgery; American Venous Forum. Earlythrombus removal strategies for acute deep venous thrombosis: clinical practice guidelines of the Society for Vascular Surgery and the American Venous Forum. J Vasc Surg 2012; 55 (5): 1449-62.

26. Kearon C, Kahn SR, Agnelli G, Goldhaber S, Raskob GE, Comerota AJ, et al. Antithrombotic therapy for venous thromboembolic disease: American College of Chest Physicians Evidence-Based Clinical Practice Guidelines (8th Edition). Chest 2008; 133 (6 Suppl): 454S-545S.

27. Jaff MR, McMurtry MS, Archer SL, Cushman M, Goldenberg N, Goldhaber SZ, et al. Management of massive and submassive pulmonary embolism, iliofemoral deep vein thrombosis, and chronict hromboembolic pul- monary hypertension: A scientific statement from the American Heart Association. Circulation 2011; 123 (16): 1788-830.

28. Vedantham S, Thorpe PE, Cardella JF, Grassi CJ, Patel $\mathrm{NH}$, Ferral H, et al. Quality improvement guidelines for the treatment of lower extremity deep vein thrombosis with use of endovascular thrombus removal. J Vasc Interv Radiol 2006; 17 (3): 435-48.

29. Vedantham S, Millward SF, Cardella JF, Hofmann LV, Razavi MK, Grassi CJ, et al. Society of Interventional Radiology. Society of Interventional Radiology position statement: treatment of acute iliofemoral deep vein thrombosis with use of adjunctive catheter-directed intrathrombus thrombolysis. J Vasc Interv Radiol 2006; 17 (4): 613-6.

30. Hartung O, Benmiloud F, Barthelemy P, Dubuc M, Boufi M, Alimi YS. Late results of surgical venous thrombectomy with iliocaval stenting. J Vasc Surg 2008; 47 (2): 381-7.

31. Hartung O, Otero A, Boufi M, De Caridi G, Barthelemy $\mathrm{P}$, Juhan $\mathrm{C}$, et al. Mid-term results of endovascular treatment for symptomatic chronic nonmalignant iliocaval venous occlusive disease. J Vasc Surg 2005; 42 (6): 1138-44.

32. Erdoes LS, Ezell JB, Myers SI, Hogan MB, LeSar CJ, Sprouse LR 2nd. Pharmacomechanical thrombolysis for phlegmasia cerulea dolens. Am Surg 2011; 77 (12): 1606-12. 